\title{
Correlation of Tumor and Peritumoral Edema Volumes with Survival in Patients with Cerebral Metastases
}

\author{
JOHANNES KERSCHBAUMER ${ }^{1}$, MARLIES BAUER ${ }^{1}$, MARINA POPOVSCAIA ${ }^{2}$, \\ ASTRID E. GRAMS ${ }^{3}$, CLAUDIUS THOMÉ ${ }^{1}$ and CHRISTIAN F. FREYSCHLAG ${ }^{1}$ \\ Departments of ${ }^{1}$ Neurosurgery, ${ }^{2}$ Medical Statistics, Informatics and Health Economics and \\ ${ }^{3}$ Neuroradiology, Medical University of Innsbruck, Innsbruck, Austria
}

\begin{abstract}
Background: Surgical resection in combination with radiotherapy in selected cases remains the best option for patients with cerebral metastases. Postoperative relapse of brain metastases occurs frequently and can be reduced by postoperative whole-brain radiotherapy (WBRT). Continuous spread of tumor cells from the primary lesions is debated as a cause of recurrence. It is well known that in gliomas, infiltration takes place within the surrounding edema. Obviously, most brain metastases are usually associated with peritumoral edema, which may act as an indicator of infiltration and more aggressive tumor biology. Therefore, we aimed to investigate the correlation of tumor and edema volumes with overall survival in patients with cerebral metastases. Patients and Methods: A total of 143 patients diagnosed with brain metastasis (male:female $=1.1: 1$ ) who underwent surgical resection were included retrospectively in this analysis. Clinical data were retrieved from electronic patient files. The volumes of tumor and edema calculated by manual delineation. The ratio of edema to tumor volume was calculated, leading to dichotomization of the patients. Results: The median tumor volume was $20.1 \mathrm{cc}$ (range $=0.8$ $90.8 \mathrm{cc}$ ) and the median volume of edema $49.5 \mathrm{cc}$ (range $=0$ $179.9 \mathrm{cc}$ ). The volume of metastases did not significantly correlate with overall survival. The ratio of edema to tumor volume was also not a prognostic factor in terms of overall survival. Only surgical resection, preoperative recursive partitioning analysis class, and postoperative addition of $W B R T$, as well as female sex, demonstrated beneficial effects. Conclusion: The extent of edema surrounding cerebral metastases does not appear to influence overall
\end{abstract}

Correspondence to: Christian F. Freyschlag, MD, Department of Neurosurgery, Medical University of Innsbruck, Anichstrasse 35, 6020 Innsbruck, Austria. Tel: +43 51250427452, Fax: +43 51250427453, e-mail: christian.freyschlag@i-med.ac.at

Key Words: Brain metastases, edema, 3D volumetry, overall survival. survival in patients suffering from brain metastases, although it seems to be responsible for most of the patients' symptoms. The hypothesis that the extent of edema was disadvantageous concerning survival was supported by our data.

Cerebral metastases (CMs) from solid cancer types are usually a sign of late-stage disease. They have been associated with high morbidity and mortality. Historically, most of these lesions have been treated by radiotherapy [focal or whole-brain radiotherapy (WBRT)] given the fact that most systemic chemotherapies are not able to overcome the blood-brain barrier. The role of neurosurgical resection in treating CMs is limited to lesions with a severe spaceoccupying effect. Recent developments in neurosurgery, such as neuronavigation, cortical mapping and intraoperative imaging, have increased patient safety. In selected patients with a limited number of CMs, microsurgical resection has shown benefits both for overall survival (OS) and functional independence (1). It was demonstrated in a randomized controlled trial that microsurgical resection provides better results when combined with whole-brain radiotherapy (WBRT) (1). Historically, surgical resection was found to be more beneficial than stand-alone radiation therapy. The fact that brain metastases are defined as circumscript lesions facilitates intraoperative dissection from unaffected brain tissue, which can be performed easily and qualifies CMs as good targets for surgical resection. A rapid relief from neurological symptoms and significant reduction in dependence on steroids underlines the efficacy of surgical treatment. Local intervention, however, does not solve the underlying problem, namely that local recurrence occurs in $50 \%$ (2) of patients within 1 year. Additional postoperative WBRT significantly reduced relapse rates to $29 \%$ (3).

The causality for recurrence following treatment is still poorly understood. Continuous seeding from systemic foci or failure of local treatments are the most reasonable explanations. Nonetheless, it is impossible to visualize progression within the microenvironment of CMs. Intracranial relapses are found in patients in whom systemic complete 
remission was reported (4-6). Local infiltrative mechanisms have been described for the appearance and recurrence of brain metastases, proven by recent autopsy studies showing distinct infiltrative patterns immunohistochemically, with integrin-staining in up to $50 \%$ of cases (7). Although the presence of vital tumor cells has never been reported, and studies assessing multiple biopsies surrounding the resection cavity found no evidence of infiltration (8), a supra-marginal resection seems to be beneficial in terms of OS (9).

Neoplastic (and inflammatory) infiltration of brain parenchyma may account for the formation of the edematous zone surrounding these lesions (10). This radiological finding in T2 or fluid-attenuated inversion recovery (FLAIR) magnetic resonance imaging (MRI) is found regularly around CMs and was the central focus of our monocentric retrospective study. We aimed to determine whether the volume of peritumoral edema could serve as a predictive marker of OS.

\section{Patients and Methods}

Patient population. Between 1999 and 2013 more than 500 patients with CMs were treated surgically by our department. Only a subpopulation of 143 patients qualified for this study, providing histopathological results, demographic data, sufficient follow-up data and pre- and postoperative MRI scans for analysis.

These 143 patients (male: female 75:67=1.1:1), aged between 34 and 85 years, were included. Demographic data such as date of operation, age at operation, date of death and OS were identified. Furthermore, extent of resection, determined by postoperative MRI ( $<48 \mathrm{~h}$ postoperatively) and histopathological diagnoses were obtained from the patients' charts. Extent of resection was classified as gross total resection, subtotal resection or biopsy by a neuroradiologist, blinded to the outcome analysis. The volume of the metastases was calculated on axial contrast-enhanced T1 MRI sequences using an OSIRIX DICOM workstation (v4.1, 32-bit, freeware) by manual delineation of the contrast-enhanced area. The volume of the peritumoral edema was calculated as the volume of hyperintense areas on T2 or FLAIR MRI sequences, with subtraction of the volume of the metastasis.

A total of 120 patients (83\%) had further radiotherapy, most of them $(n=106,74 \%)$ were treated with WBRT, whereas only a minor proportion underwent local radiotherapy of tumor and peritumoral edema, according to the decision of our Institutional Tumor Board.

Study design. The study was performed as a retrospective monocentric analysis. Inclusion criteria were histopathologically confirmed cerebral metastasis plus availability of sufficient clinical and imaging data.

Statistical analysis. To carry out the correlation of tumor volume and associated edema, a coefficient (ratio $=\mathrm{V}_{\text {edema }} / \mathrm{V}_{\text {tumor }}$ ) was calculated and the cohort subsequently dichotomized for analysis. Cases in which the volume of edema was larger than the actual tumor volume (ratio $>1$ ) and cases in which the edema was smaller than the tumor were tested for correlation with outcome parameters. A comparison of the two groups by Cox regression and a KaplanMeier survival calculation were performed. Group differences and
Table I. Demographic characteristics of patients.

\begin{tabular}{lc}
\hline & $\mathrm{n}(\%)$ \\
\hline Patients & \\
Male & $76(53.1)$ \\
Female & $67(46.9)$ \\
Primary tumor & \\
NSCLC & $51(35.7)$ \\
Breast cancer & $19(13.3)$ \\
Melanoma & $19(13.3)$ \\
Lower GIT & $13(9.1)$ \\
RCC & $11(7.7)$ \\
CUP & $4(2.8)$ \\
SCLC & $8(5.6)$ \\
Other & $18(12.6)$ \\
Extent of resection & \\
GTR & $101(70.6)$ \\
STR & $26(18.2)$ \\
Biopsy only & $16(11.2)$ \\
\hline
\end{tabular}

CUP: Carcinoma of unknown primary; GIT: gastrointestinal tract; GTR: gross total resection; NSCLC: non small-cell lung cancer; RCC: renal cell cancer; SCLC: small-cell lung cancer; STR: subtotal resection.

correlations between variables were considered significant at a $p$ value below 0.05. Statistical analysis was performed using SPSS 21.0 (IBM Corp., Armonk, NY, USA).

\section{Results}

Based on postoperative MRI, a gross total resection was accomplished in the majority of patients $(70.6 \%)$. The most common type of primary tumor was non-small cell lung cancer (NSCLC) with 51 cases $(35.7 \%)$. Four cases of cancer of unknown primary; and 18 cases with different rare entities were excluded from the distinct subgroup analysis due to low number. The median OS ranged from less than 1 month to 116 months, with a median of 14.9 months (Table I).

Mean tumor volume was $20.1 \mathrm{cc}$, while mean edema volume was 49 in $5 \mathrm{cc}$ without significant differences between the distinct primary tumor types (Figure 1a). There was a difference in tumor volume and volume of edema for distinct histological subtypes as SCLC, breast cancer and melanoma had higher mean tumor volumes than the other entities, although failing to reach statistical significance. Metastases of cancer of the lower gastrointestinal tract and NSCLC were greater in volume than the surrounding edema. Therefore, statistical significant differences in the ratios of $\mathrm{V}_{\text {edema }}$ and $\mathrm{V}_{\text {tumor }}$ were found for the different primaries (Figure 1b). The ratio of edema to tumor volumes, however, showed no impact on OS $(p=0.38)$, neither in multivariate, nor in univariate analysis. When comparing non-small cell lung cancer (NSCLC) and SCLC, there was statistically significantly less edema in the SCLC group (Figure 1b). 

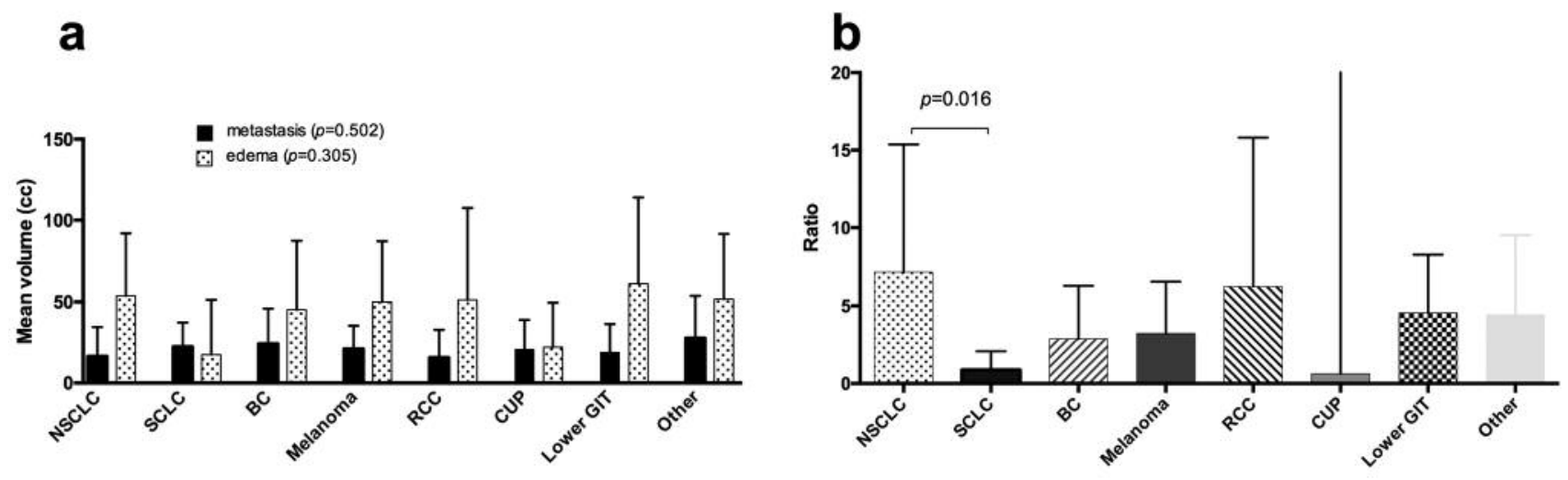

Figure 1. Tumor volume and the volume of the surrounding edema in patients with cerebral metastases from different primary tumor types. There was no significant difference in the amount of edema between the different types of primaries; however, the ratio of volume of the edema to that of the metastasis differed significantly for small-cell lung cancer (SCLC) vs. non-small-cell lung cancer (NSCLC). Data are the mean $\pm S D$ BC: Breast cancer; CUP: carcinoma of unknown primary; GIT: gastrointestinal tract; RCC: renal cell cancer.
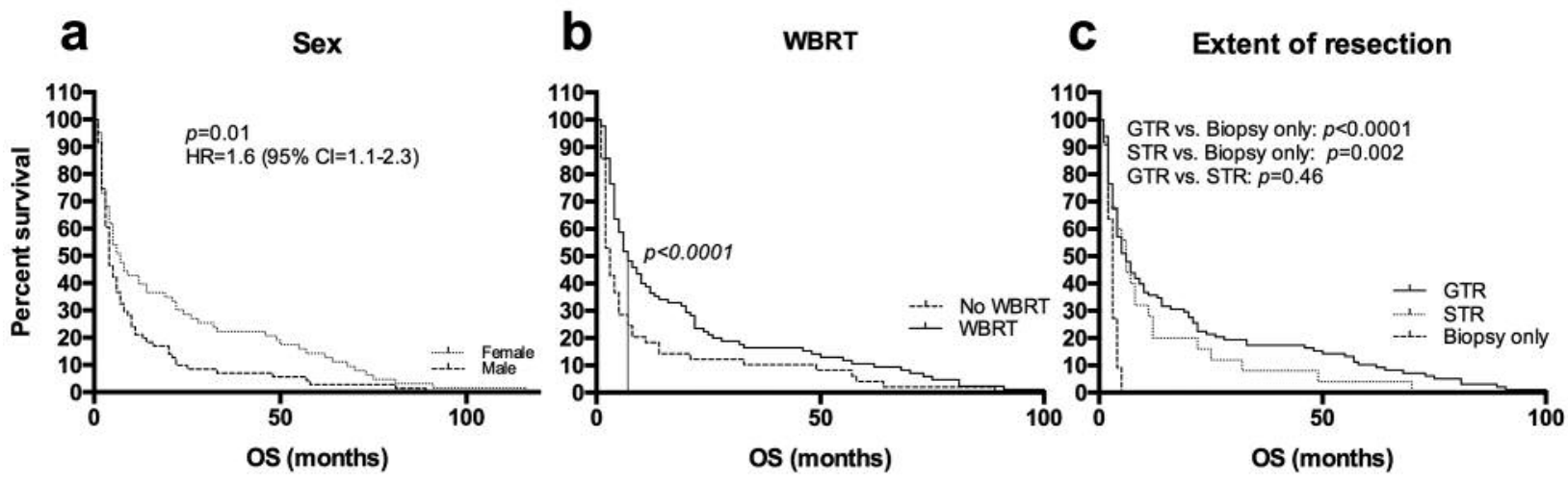

Figure 2. Prognostic factors in patients with cerebral metastases. Female sex (a) and postoperative whole-brain radiotherapy (WBRT) (b) were of significant benefit in terms of overall survival (OS), whereas residual tumor on postoperative magnetic resonance imaging was not significantly associated with shorter OS, except for patients in whom only biopsy was performed (c). GTR: Gross total resection, STR: subtotal resection.

Most patients $(n=81)$ operated on for CMs presented a singular metastasis in the brain. However, there were also patients included with up to eight metastases who were subject to surgical treatment if they were severely symptomatic due to one of the metastatic lesions (28 patients with two metastases, eight patients with three and four metastases each, one patient with five and one with eight metastases and one patient with six). The OS of patients with multiple CMs was equivalent to that of patients with single CMs when WBRT was administered after resection $(p=0.63)$. The extent of resection was found to be significant in terms of OS $(p=0.001)$, but there was no difference between those who underwent gross total resection and those who underwent subtotal resection ( $p=0.24)$ (Figure 2c).

A total of $76.9 \%$ of the patients were classified as recursive partitioning analysis (RPA) class 2 , whereas only
14.7\% had no extracranial disease manifestation (RPA class 1). Moreover $8.4 \%$ of the patients were operated on for symptomatic brain metastasis, despite presenting in a poor general condition (RPA class 3 ). Lower RPA class was also an independent predictor of prolonged OS $(p<0.001)$.

In multivariate analysis, the age at time of surgery was statistically significant as a prognostic factor for OS $(p<0.001)$; furthermore, female sex was a positive predictor of OS ( $p=0.01$, Figure 2a).

\section{Discussion}

Our study revealed no correlation of the extent of edema with OS in surgicall-treated patients with cerebral metastases. As a result of the improved survival in modern medical oncology, neurosurgeons and neuro-oncologists are 
facing an increasing number of patients with CMs. As medical treatment options in the brain are limited, focal therapies such as surgery and radiotherapy remain state-ofthe art treatment for brain metastasis. A proof of glioma-like infiltrative behavior of CMs would question the value of a standalone focal therapy and support the necessity for interdisciplinary treatment strategies.

Microsurgical resection is considered superior in large lesions with mass effect and may restore neurological deficits quickly. It has been shown that surgical resection reduces the use of steroids and establishes a good quality of life (1). Used alone, however, it did not prevent prompt recurrence (3). Radiosurgical treatment provides similar rates of local control and immediate relapse in patients with CMs compared to standalone surgical resection $(3,11)$. WBRT cannot deliver equivalent irradiation doses to a single metastasis, but it is the only modality that may influence infiltrating tumor cells in the brain parenchyma, not visualized by MRI. Due to the prolonged survival of patients after WBRT, long-term adverse events are seen more frequently. Predominately, side-effects are recognized as neuropsychological impairment, fatigue and decline of cognitive functions (12). We demonstrated that additional WBRT in our patients led to a significantly prolonged OS, even in cases of multiple CM or incomplete resection.

Both the cause and the importance of peritumoral edema in patients with CMs remain uncertain. In contrast to gliomas, with their distinct infiltration within the T2hyperintense shade (13), metastases are thought to cause pure vasogenic edema due to the mass effect or secretion of inflammatory cytokines. Even if direct infiltration of the surrounding brain parenchyma by metastases has not been proven by the detection of vital tumor cells $(8,14)$, there are many theories of infiltration as a source of local relapse. Yoo et al. (8) and Kamp et al. (9) were able to significantly minimize local recurrences by resecting $2-5 \mathrm{~mm}$ beyond the tumor margin within the edematous zone. However, both trials failed to detect vital tumor cells within histopathological investigation of the supramarginal resected tissue. Recently, autopsy brain specimens of patients with known CMs were examined via indirect staining for integrins and other adhesion molecules to determine infiltration patterns. Berghoff et al. were able to differentiate between diffuse and vascular co-opted infiltration in $50 \%$ of their specimens (7). The same group of investigators had contradictory findings, correlating lesser edema around CMs with worse outcome and suspecting different types of infiltration to account for different extents of edema (10). In their study, axial diameters of edema in patients with CMs were compared to infiltrative behavior (reported intraoperatively) and OS. The findings in our study did not confirm the hypothesis that edema is a predictor of OS. We were not able to identify any correlation between the extent of the peritumoral edema and OS, neither in the whole cohort, nor in the sub-analysis of different primaries.

Our study is limited by the number of heterogeneous primaries included in the analysis. The most common types of solid cancer, which preferentially metastasize to the brain, however, were adequately represented.

\section{Conclusion}

This retrospective analysis of patients with brain metastases demonstrates that the extent of peritumoral edema had no influence on OS in patients harboring CMs. Beneficial factors for achievement of prolonged OS were extent of resection, RPA-classification, patient's age and female sex.

\section{Conflicts of Interest}

The Authors declare they have no conflict of interest.

\section{References}

1 Patchell RA, Tibbs PA, Walsh JW, Dempsey RJ, Maruyama Y, Kryscio RJ, Markesbery WR, Macdonald JS and Young B: A randomized trial of surgery in the treatment of single metastases to the brain. New Engl J Med 322: 494-500, 1990.

2 Baumert BG, Rutten I, Dehing-Oberije C, Twijnstra A, Dirx MJ, Debougnoux-Huppertz RM, Lambin P and Kubat B: A pathology-based substrate for target definition in radiosurgery of brain metastases. Int J Radiat Oncol Bio Phys 66: 187-194, 2006.

3 Kocher M, Soffietti R, Abacioglu U, Villa S, Fauchon F, Baumert BG, Fariselli L, Tzuk-Shina T, Kortmann RD, Carrie C, Ben Hassel M, Kouri M, Valeinis E, van den Berge D, Collette S, Collette L and Mueller RP: Adjuvant whole-brain radiotherapy versus observation after radiosurgery or surgical resection of one to three cerebral metastases: results of the EORTC 22952-26001 study. J Clin Oncol 29: 134-141, 2011.

4 Kastritis E, Efstathiou E, Gika D, Bozas G, Koutsoukou V, Papadimitriou C, Pissakas G, Dimopoulos MA and Bamias A: Brain metastases as isolated site of relapse in patients with epithelial ovarian cancer previously treated with platinum and paclitaxelbased chemotherapy. Int J Gynecol Cancer 16: 994-999, 2006.

5 Manapov F: Central nervous system relapse continues to be a therapeutic challenge in extensive disease small-cell lung cancer patients with initial symptomatic brain metastases and good response to chemoradiotherapy. J Neurooncol 98: 349-355, 2010.

6 Morizawa Y, Momose H, Toyoshima Y, Takada S, Fujimoto K and Oyama N: Brain metastasis which appeared during complete response of lung metastatic lesion by sunitinib treatment in a patient with renal cell carcinoma. Hinyokika Kiyo 59: 791-793, 2013.

7 Berghoff AS, Rajky O, Winkler F, Bartsch R, Furtner J, Hainfellner JA, Goodman SL, Weller M, Schittenhelm J and Preusser M: Invasion patterns in brain metastases of solid cancers. Neuro Oncol 15: 1664-1672, 2013.

8 Yoo H, Kim YZ, Nam BH, Shin SH, Yang HS, Lee JS, Zo JI and Lee SH: Reduced local recurrence of a single brain metastasis through microscopic total resection. J Neurosurg 110: 730-736, 2009. 
9 Kamp MA, Dibue M, Niemann L, Reichelt DC, Felsberg J, Steiger HJ, Szelenyi A, Rapp M and Sabel M: Proof of principle: supramarginal resection of cerebral metastases in eloquent brain areas. Acta neurochir (Wien) 154: 1981-1986, 2012.

10 Spanberger T, Berghoff AS, Dinhof C, Ilhan-Mutlu A, Magerle M, Hutterer M, Pichler J, Wohrer A, Hackl M, Widhalm G, Hainfellner JA, Dieckmann K, Marosi C, Birner P, Prayer D and Preusser M: Extent of peritumoral brain edema correlates with prognosis, tumoral growth pattern, HIF1a expression and angiogenic activity in patients with single brain metastases. Clin Exp Metastasis 30: 357-368, 2013.

11 Scoccianti $\mathrm{S}$ and Ricardi U: Treatment of brain metastases: review of phase III randomized controlled trials. Radiother Oncol 102: 168-179, 2012.

12 Tallet AV, Azria D, Barlesi F, Spano JP, Carpentier AF, Goncalves $\mathrm{A}$ and Metellus P: Neurocognitive function impairment after whole brain radiotherapy for brain metastases: actual assessment. Radiat Oncol 7: 77, 2012.
13 Miwa K, Shinoda J, Yano H, Okumura A, Iwama T, Nakashima $\mathrm{T}$ and Sakai N: Discrepancy between lesion distributions on methionine PET and MR images in patients with glioblastoma multiforme: insight from a PET and MR fusion image study. J Neurol Neurosurg Psychiatry 75: 1457-1462, 2004.

14 Li B, Yu J, Suntharalingam M, Kennedy AS, Amin PP, Chen Z, Yin R, Guo S, Han T, Wang Y, Yu N, Song G and Wang L: Comparison of three treatment options for single brain metastasis from lung cancer. Int J Cancer 90: 37-45, 2000.

Received December 12, 2016

Revised January 17, 2017

Accepted January 20, 2017 